TITLE:

\title{
Awe or horror: differentiating two emotional responses to schema incongruence
}

$\operatorname{AUTHOR}(\mathrm{S})$ :

Taylor, Pamela Marie; Uchida, Yukiko

CITATION:

Taylor, Pamela Marie ...[et al]. Awe or horror: differentiating two emotional responses to schema incongruence. Cognition and Emotion 2019, 33(8): 1548-1561

\section{ISSUE DATE:}

2019-11-17

URL:

http://hdl.handle.net/2433/244112

\section{RIGHT:}

This is an Accepted Manuscript of an article published by Taylor \& Francis in 'Cognition and Emotion' on 2019, available online: https:/ www.tandfonline.com/10.1080/02699931.2019.1578194: The full-text file will be made open to the public on 06 Feb 2020 in accordance with publisher's 'Terms and Conditions for Self-Archiving'; この論文は出版社版でありま せん。引用の際には出版社版をご確認ざ利用ください。; This is not the published version. Please cite only the published version. 
6

$7 \quad{ }^{1}$ Kyoto University, Graduate School of Human and Environmental Sciences, Yoshida-

8 Honmachi, Sakyo-ku, Kyoto 606-8501, Japan

$9 \quad{ }^{2}$ Kokoro Research Center, Kyoto University, Kyoto, Japan

$10 *$ Corresponding author

11 email: teira.mari.24a@st.kyoto-u.ac.jp

12

13

14 


\section{Abstract}

2 Experiences that contradict one's core concepts (e.g. of the world, people, the self)

3 elicit intense emotions. Such schema incongruence can elicit awe, wherein experiences that

4 are too vast to understand with existing cognitive schemata cause one to feel that schemata

5 should be updated [i.e., a "need for cognitive accommodation” (NFA); Keltner \& Haidt,

6 2003]. However, other emotional responses to schema incongruence, such as horror, have not

7 been investigated.

8 The current studies compared awe and horror to investigate if they are distinct

9 schema-incongruent emotional responses. Study 1 observed significant differences between

10 awe and horror in cognitive appraisals (e.g., certainty, legitimacy), indicating several areas of

11 dissimilarity. Study 2 found evidence that awe and horror are both responses to schema-

12 incongruence, as schema incongruence and NFA were salient in awe and horror, but not a

13 contrast emotion. However, awe and horror were elicited by different types of schema

14 incongruence: awe by spiritual vastness, horror by extremity. Awe-eliciting experiences also

15 appeared to be easier to assimilate than horrifying experiences, as NFA and uncertainty were

16 significantly lower in awe than in horror. Differences in the functions of horror and awe are

17 also discussed.

18 Word count: 185

19 Keywords: awe, horror, emotional appraisals, cognitive schema 
“[L]ike it or not, Auschwitz expands the universe of consciousness no less than landing on the moon.” - Miller, 2004

\section{Introduction}

Emotions are shaped by cognitive schemata. Schemata, patterns of cognition that organize knowledge and conceptual relationships (Fiske \& Linville, 1980), are developed via experience and help us quickly appraise events. In turn, these appraisals elicit emotions

9 (Smith \& Ellsworth, 1985). If a situation is consistent with one's cognitive schemata, it elicits an emotion that motivates adaptive behavioural responses (Keltner \& Gross, 1999) and reinforces the schemata that guided it (Clore \& Gasper, 2000). But what is the emotional response to experiences that are incommensurable with one’s existing schemata?

This paper proposes that events and phenomena that violate mental representations of the world elicit “schema-incongruent emotions” that motivate not just behavioural responses, but also cognitive restructuring of the categories through which one interprets the world, i.e., cognitive accommodation (Piaget, 1950). Awe is one such schema-incongruent emotion

17 (Keltner \& Haidt, 2003) and horror is another. Although some view horror as a subtype of awe (Gordon et al., 2017; Shiota, Keltner, \& Mossman, 2007), the current studies provide evidence that they are different emotional responses.

Although the emotional components of awe have been investigated (e.g., Bonner \&

21 Friedman, 2011; Shiota et al., 2007), horror has yet to be investigated as a natural emotion[insert footnote 1 here]. However, horror appears to exhibit the five components of emotion (Scherer, 2005): Darwin (1872) described horror's facial expression and biophysiological symptoms, PTSD researchers[insert footnote 2 here] (e.g., Janoff-Bulman, 
1 1992) and philosophers (Carroll, 1990) have detailed the subjective experience of horror,

2 survival psychology has described horror's action tendencies (Leach, 1994) and linguistic

3 analysis has identified appraisals that distinguish horror from other fear states (Wierzbicka,

4 1988).

\section{Emotional responses to schema incongruence}

Cognitive schemata are generalised beliefs about the self and the world that help

7 humans efficiently understand and categorise information and experiences. They operate

8 automatically and can be activated by external stimuli without conscious awareness (Nisbett

9 \& Wilson, 1977; Taylor \& Fiske, 1978). Schemata are resilient against disconfirmation:

10 information that does not fit is usually discounted, ignored, reappraised or revised to be consistent with current schemata (Lodge \& Taber, 2000). Such “cognitive assimilation” is efficient and maintains a consistent worldview, so it is the default human informationprocessing style (Piaget, 1950).

However, experiences that cannot be explained with existing schemata are often relevant to survival (e.g., signal changes to vital environmental or social resources). Thus, the adaptive cognitive response to schema incongruence is to update one's schemata accurately,

17 without schema-driven alteration (Diamond \& Zoladz, 2016). This is "cognitive accommodation”, which entails bottom-up, stimulus-driven information processing (as opposed to the top-down, schema-driven information processing of assimilation; Bless \&

20 Fiedler, 2006; Fiedler \& Bless, 2000). The difference between assimilation and accommodation can be illustrated with the example of the 9/11 terrorist attacks, in which terrorists flew two planes into the World Trade Centre in New York. Immediately after the

23 first plane hit, people assimilated the news by interpreting the event as an accident, which

24 was consistent with the schemata it activated (e.g., airline crashes). However, the second 
1 plane strike invalidated the accident interpretation and forced people to accommodate their

2 understanding of the world to incorporate a new reality (Yum \& Schenck-Hamlin, 2005). Emotional responses to schema-incongruence may facilitate cognitive accommodation

4 by increasing neurocognitive plasticity (Diamond, Campbell, Park, Halonen, \& Zoladz, 2007)

5 and weakening the attitudes, beliefs and assumptions that would otherwise facilitate

6 assimilation (Dalgleish \& Power, 2004; Lodge \& Taber, 2000). This is experienced as a

7 “need for cognitive accommodation" (NFA): the perception that one cannot assimilate an

8 experience into existing schemata and should therefore modify one’s understanding of the

9 world to make sense of it (Keltner \& Haidt, 2003). NFA shifts attention away from internal

10 knowledge structures and toward the external elicitor, privileging data-driven perceptual

11 processing over meaning-making conceptual processing (Bless \& Fiedler, 2006). In

12 summary, schema-incongruent information both activates and violates a schema, which

13 elicits intense emotions that increase cognitive plasticity and bottom-up processing to

14 facilitate schematic revision.

15 Two types of schema-incongruence: Vastness and extremity

"Schema incongruence" is how much an experience or entity deviates from "the self's

17 ordinary level of experience or frame of reference" (Shiota et al., 2007, p. 945). Because schemata are “cognitive manifestations of psychological needs” (e.g., security, interpersonal belonging; McCann \& Pearlman, 1990), they are sensitive to changes in one’s physical and social environments that affect one's ability to satisfy these needs. Different types of schema incongruence elicit different responses, depending on what signals they give about the environment.

Some types of schema-incongruity - such as vastness - signal new opportunities to

24 meet one’s needs (e.g., abundant nature, extraordinary people, spiritual forces). Vast

25 phenomena exist on a scale beyond full human comprehension and typically elicit awe 
1 (Keltner \& Haidt, 2003), which is associated with increased schematic flexibility that helps

2 one capitalise on the opportunities signalled by vastness (Armstrong \& Detweiler-Bedell,

3 2008; Shiota et al., 2007). Awe-eliciting vastness causes categories to become more inclusive

4 (Shiota et al., 2007), less egocentric (Piff, Dietze, Feinberg, Stancato, \& Keltner, 2015) and

5 more metaphysically-oriented (Van Cappellen \& Saroglou, 2012) and can increase

6 perceptions of purpose, meaning and order in the world (Valdesolo \& Graham, 2014).

Conversely, another type of schema-incongruity - extremity - signals that vital

8 resources, relationships or assumptions have become unviable or dangerous and one must

9 adapt to meet one's needs. Similar to biological extremity (i.e., conditions too abnormal to

10 acclimate to and therefore necessitate adaptation; Gutschick \& BassiriRad, 2003), extremity

11 is constituted by events or behaviors that deviate too much from basic world assumptions to

12 be assimilated, and instead require schematic accommodation. Extremity exceeds the limits

13 of comprehensibility or presumed possibility and is thus evaluated as "unthinkable” and

14 elicits appraisals associated with horror, such as, “I didn’t think that something like this could

15 happen” (Wierzbicka, 1988). For example, although deception and aggression are considered

16 morally wrong, they are understandable: "Most people can conceive of themselves lying,

17 stealing, breaking a promise, robbing a store, hitting someone, even killing”. However, extreme deception or aggression "baffles understanding" and "stretch[es] our imaginations, our understanding of what human beings are capable of” because it is “conduct that one cannot conceive of oneself engaging in” (Singer, 2004, p. 195-196). conceptual expansiveness that violates schematic scale expectations and reveals the limits of one’s knowledge. For example, someone whose experience with trees has been limited to

$2412 \mathrm{~m}$ tall trees will have their concept of nature's scale violated by seeing a $110 \mathrm{~m}$ tall tree,

25 which will motivate adjustment of that schema's spatial criteria. Because this schema 
1 incongruity does not contradict other defining schematic traits, this accommodation is not

2 problematic. In contrast, extremity typically contradicts or negates core schematic traits. In

3 extremity (i.e., excessive conceptual distance from schematic norms), an entity (often a

4 person) exhibits a trait or behavior to a disproportionate degree that violates basic schematic

5 criteria for category inclusion. For example, the schema of "parent” includes both protecting

6 and disciplining one's child. But a parent whose extreme "discipline” contravenes

7 “protection” (e.g., starving a child as punishment) violates the "parent” schema. To

8 accommodate this attribute-level schema incongruity, either the perpetrator must be

9 dissociated from the category (e.g., create new schema of "unfit parents") or the "parent”

10 schemata must change to acknowledge that all parents can act similarly.

\section{Awe and horror are distinct emotional responses to schema incongruence}

We propose that awe and horror are two different emotional responses to schema incongruence. Schema-incongruent emotions are a subcategory of "knowledge emotions"

14 (e.g., surprise, interest, confusion), which are elicited by challenges to one's thoughts and 15 knowledge (Silvia, 2010). We propose that schema-incongruent emotions are distinct among knowledge emotions in being elicited by global challenges to one's schematic structures (i.e.,

17 how one's worldview is organized), rather than local challenges to the content of one's knowledge. Whereas trivial aberrations or novelties elicit surprise, confusion or interest, awe and horror are elicited by contradictions of core schemata (e.g., moral values). speculated that horror is a combination of fear and awe (Shiota et al., 2007) or a subtype of awe (Gordon et al., 2017, Study 2b). However, empirical and theoretical research indicates

23 that awe and horror are different emotional responses to different types of schema

24 incongruence. Awe is elicited by entities and actions that enhance and strengthen systems of meaning (Yaden, Haidt, Hood, Vago, \& Newberg, 2017), such as art and moral excellence 
1 (Shiota et al., 2007). In contrast, horror elicitors, such as catastrophe and cruelty, undermine

2 systems of meaning (Kristeva, 1982). Thus, they have opposite effects on basic world

3 assumptions: horror "shatters" them (Janoff-Bulman, 1992), but awe affirms or expands them

4 (Van Cappellen, Saroglou, Iweins, Piovesana, \& Fredrickson, 2013).

Horror may sometimes be conflated with awe because both can involve fear (Gordon et al., 2017; Piff et al., 2015). However, the fear in awe and horror differ in several aspects.

7 First, fear is central to horror, but peripheral and non-essential to awe (Bonner \& Friedman,

8 2011; Keltner \& Haidt, 2003). Second, whereas horror is a response to physical or existential

9 damage (Cavell, 1979), the fearful aspects of awe are appraised as benign (Bonner \&

10 Friedman, 2011). Third, the fear in awe reflects respect or deference towards power (Keltner

11 \& Haidt, 2003), but in horror is antagonistic to the elicitor and rejects it as abhorrent and

12 unnatural (Carroll, 1990; Kristeva, 1982). Fourth, horrified fear is a response to the realization that actual harm is currently occurring or has occurred (Cavell, 1979), but fear in awe is felt towards the hypothetical harm a powerful elicitor could cause in the future (Kant, 1790/1951).

Awe and horror also have different cognitive effects. Awe can expand ontological schemata beyond the material world, leading to metaphysical inferences (Valdesolo \& Graham, 2014), feelings of personal connection with higher powers (Piff et al., 2015) and ingroup members (Krause \& Hayward, 2015) and a reduced sense of a personal self (Yaden et al., 2017). In contrast, horrifying experiences disrupt schemata related to safety, trust, power, self-esteem and/or intimacy (Lisa McCann, Sakheim, \& Abrahamson, 1988).

The Present Research

The goal of this research is to investigate if awe and horror are different emotional responses to schema-incongruent experiences. To test this, we investigated if they differ in cognitive appraisals (Study 1) and elicitors (Study 2). Additionally, to test if awe and horror 
1 are both responses to schema-incongruence, we investigated if schema-incongruence and

2 NFA were greater in awe and horror than in the schema-congruent emotion of contentment

3 (Study 2).

\section{Study 1: Cognitive appraisals in awe and horror}

Study 1 compared cognitive appraisals in awe and horror to investigate how they are

6 different and similar.

Hypothesis 1: because horror is aversive, Horror[insert footnote 3 here] will have

8 lower attention-related appraisals (e.g., focusing on the elicitor) than Awe.

Hypothesis 2: because horror is associated with survival situations, Horror will have

higher appraisals of goal-path obstacles and anticipated effort than Awe.

Hypothesis 3: because horror is associated with interpersonal conflict, Horror will

have higher appraisals of external human agency and lower appraisals of legitimacy and pleasantness than Awe.

Hypothesis 4: because we assume that both are schema-incongruent emotions, we

and personal agency, and similarly high appraisals of situational agency[insert footnote 4

17 here].

Method

\section{Participants}

Two-hundred-and-nineteen participants recruited on Mechanical Turk completed a

21 "survey on emotional experiences” for US\$1.25. Participants were required to be U.S.-born

22 native English speakers who were at least 18 years old and had not lived outside the U.S. for more than two years. Participants exhibiting any of the following were excluded from analysis: failed an attention check item, did not follow directions on open-response questions 
1 (e.g., did not write about a specific event), exhibited answer straight-lining (low response

2 variance, $s d<.50$ for all Likert responses) or rated feeling the target emotion "not at all".

3 Thirty-one participants' data were excluded, leaving 188 participants for analysis[insert

4 footnote 5 here $\left(M_{\text {age }}=36.80, S D_{\text {age }}=11.17\right.$, Range $=20-71$; 96 females, 89 males, 2 other;

5 76.4\% European American, 9.9\% African American, 1.1\% Latino, 3.2\% Asian American,

$6 \quad$ 0.5\% South Asian, 8.5\% mixed race). A post-hoc power analysis[insert footnote 6 here] using

7 G*Power (Faul, Erdfelder, Lang, \& Buchner, 2007) indicated 92\% power to detect medium-

8 sized effects $(d=.5)$ in independent t-tests with a .05 two-tailed type 1 error probability.

Procedure

After providing consent, participants were randomly assigned to the Awe condition or

the Horror condition and asked to recall a personal experience of the target emotion, defined as:

“[Awe/Horror] is an emotion felt towards something vast[insert footnote 7 here] that you can't fully understand at the time, in which..."

Awe Condition: “...you feel someone or something is amazing or sublime.”

Horror Condition: “...someone or something was harmed or damaged.”

Participants responded to the prompt via nine open-response questions adapted from

Smith \& Ellsworth (1985; e.g., “What happened to make you feel awe/horror?”). Next,

participants rated cognitive and emotional appraisals of the target experience.[insert footnote

8 here] Lastly, they provided demographic information and were debriefed.

Measures

Cognitive appraisals. Participants rated 23 items measuring nine appraisal domains of the target experience adapted from Smith and Ellsworth (1985) using a nine-point Likert 
1 means)[insert footnote 9 here]. The items for each domain were averaged to form aggregate

2 variables (see Table 1).

3

Manipulation check. Participants rated how much they felt awe and horror during the

4 situation (presented among twenty emotion items) on a 7-point scale (1=“not at all”,

5 7=“extremely”).

$6 \quad$ Results

7

8

9

\section{Manipulation check}

Welch's t-tests showed that awe was higher in the Awe condition and horror was higher in the Horror condition (see Table 1). Awe and horror were uncorrelated in both conditions, $r s \leq|.07|, p s \geq .51$.

\section{Appraisal dimensions}

Welch's t-tests showed Awe and Horror differed in cognitive appraisals (see Table 1). Consistent with Hypotheses 1-3, appraisals of attentional activity and fairness were greater in Awe, appraisals of external human agency, goal-path obstacles and anticipated effort were greater in Horror, and appraisals of situational agency were equal between conditions. However, contrary to Hypothesis 4, personal agency and certainty were significantly higher in Awe than in Horror.[insert footnote 10 here]

$$
\text { [Table } 1 \text { near here] }
$$

\section{Discussion}

Emotion ratings for awe and horror were uncorrelated and between-condition meandifferences had large effect sizes, supporting our general hypothesis that horror and awe are different emotional experiences.

Awe and Horror differed in eight of nine appraisal dimensions. Horror had lower appraisals of self-agency and certainty, which, combined with appraisals of greater goal-path 
1 obstacles and anticipated effort, indicate coping anxiety, possibly because horrifying

2 situations have physical or cognitive demands that exceed one’s abilities (Leach, 1994). In

3 contrast, the low appraisals of anticipated effort and goal-path obstacles in Awe may indicate

4 that the physical world and its constraints were less salient (e.g., self-transcendence; Yaden,

5 Haidt, et al., 2017).

Contrary to Hypothesis 4, certainty was higher in Awe than in Horror. Other research

7 similarly found that certainty was higher in positive awe than negative "threat-based awe"

8 (Gordon et al., 2017), but the effect size observed here is three times larger. This could

9 indicate that horror involves greater NFA than awe or that horrific NFA is more difficult to

10 resolve (Dalgleish \& Power, 2004). It is also possible that spontaneous meaning-making

11 helps accommodate schema-incongruence in awe (e.g. Valdesolo \& Graham, 2014) but not in

12 horror.

13 Situational agency was the only appraisal dimension with no between-condition

14 difference. However, situational agency appraisals probably reflect different attribution

15 targets in the two emotions (Brewin \& Shapiro, 1984). In Awe, situational agency may reflect

16 feelings of self-transcendence or a "small self” (Piff et al., 2015). In contrast, because horror

17 is elicited by harm and involves blame-related appraisals (external human agency, low

18 legitimacy), situational agency in horror may reflect hostility and blame (Feigenson, 2002) or

19 feelings of personal helplessness or dissociation (Brewin \& Holmes, 2003).

One limitation of Study 1 was that it only compared awe and horror against each

21 other. Thus, we can discuss how they differ from each other, but not how they might be

22 similar relative to schema-congruent emotions. Therefore, Study 2 included a contrast

23 condition. 


\section{Study 2: Vastness and NFA in awe and horror}

2

3

4 6 (Berenbaum, 2002). than Contentment.

\section{Method}

\section{Participants}

We propose that both awe and horror are elicited by schema incongruence and result in NFA. To test if schema incongruence and NFA are greater in awe and horror than a schema-congruent emotion, Study 2 contrasted awe and horror with contentment, which is elicited by schema congruent experiences, such as the satisfaction of basic needs and goals

Hypothesis 1: Awe and Horror will have significantly more schema incongruence

Hypothesis 2: Awe and Horror will have significantly more NFA than Contentment. An additional exploratory purpose of this study was to investigate what types of experiences elicit awe and horror. To do so, a qualitative analysis was performed on participants’ written descriptions of awe and horror. Because coding categories were created using inductive analysis, we had only one hypothesis about elicitors:

Hypothesis 3: Horror will be elicited by harm more frequently than Awe.

One-hundred-and-eighty-one participants recruited on Mechanical Turk participated for US\$2.00. Participant requirements and exclusion criteria were identical to Study 1, resulting in the exclusion of 47 participants' data[insert footnote 11 here], leaving 134 for analysis $\left(\right.$ females $=81$, males $=53 ; M_{\text {age }}=38.55, S D_{\text {age }}=12.21$, Range $=20-64 ; 76.1 \%$ European American, 7.5\% African American, 5.2\% East Asian American, 2.2\% South Asian or Indian, 1.5\% Latino or Hispanic, 0.7\% Middle Eastern, 0.7\% Native American, 5\% mixed ethnicity, $0.7 \%$ chose not to answer). A post-hoc power analysis[insert footnote 12 here] using $\mathrm{G}^{*}$ Power indicated $93 \%$ power to detect medium-sized effects in chi-square tests $(\varphi$ $=.3$ ) with $\alpha=.05$ and $95 \%$ power to detect medium-sized effects in omnibus within-factors 
1 ANOVAs $\left(\eta^{2}=.06\right)$ using three repeated measures with $\alpha=.05$ and post-hoc pairwise

2 comparisons with Bonferroni-adjusted $\alpha=.017(d=.5)$.

3

4

Procedure

A within-subjects design was used with three conditions (Awe, Horror, Contentment) randomly counterbalanced. After providing consent, participants were asked to "vividly recall a personal experience of [awe/horror/contentment] (not including experiences of fictional products, e.g., movies, books)” and write 3-5 sentences about it. After writing about the first target emotion, they rated its schema incongruence and NFA. This process was repeated for the second and third emotions in turn. Lastly, participants provided demographic data and were debriefed.

To explore what elicits awe and horror, written responses for the Awe and Horror conditions were coded for elicitor categories. Categories were created via inductive coding by the first author. Two hypothesis-blind coders applied dichotomous (applicable, not applicable), non-mutually-exclusive elicitor codes to scenarios. The elicitor categories were: “another person(s)”[insert footnote 13 here], “event”, “nature”, “human-made objects”, “location or physical surroundings”, “information”[insert footnote 14 here] and “animals”. Additionally, to differentiate horror from negative threat-based awe, scenarios were coded with mutually-exclusive themes of "harm”, “death” and "threat without harm”, as well as a non-mutually exclusive theme of "silver linings" (i.e., something positive occurring during something negative). Thirty-four percent of scenarios[insert footnote 15 here] were coded by both coders and initial Krippendorf alphas ranged .69-1.00, $M=.81$.[insert footnote 16 here]

Coding disagreements were resolved via three-way discussion with the first author.

3 Measures

Schema Incongruence and NFA. Items measuring schema incongruence and NFA were based on previous theoretical and empirical descriptions of schema incongruence in awe 
1 and horror (Keltner \& Haidt, 2003; Janoff-Bulman, 1992; Schurtz et al., 2012; Shiota et al.,

2 2007; Piff et al., 2015). Because current theory asserts that schema incongruence causes NFA

3 (Janoff-Bulman, 1992; Keltner \& Haidt, 2003), schema incongruence items measured how

4 much elicitors deviated from normality, and NFA items measured cognitive reactions to such

5 deviations. All items were rated on five-point Likert scales.

Schema Incongruence: Participants rated the schema incongruence of the eliciting experience in relative and absolute terms (see Tables 3 and S2). Relative incongruence measured deviance from "things you normally experience in everyday life" (-2="much less than normal”, $0=$ "the same as normal”, $+2=$ =“much greater than normal”; remapped to $1-5$ scale for analysis). Absolute incongruence measured the applicability of general schemadeviance qualities (1=“does not describe it at all”, 5=“describes it extremely well”). assimilating the experience (1=“does not describe it at all”, 5=“describes it extremely”), belief/value contradiction (1=“very much contradicted”, 3=“neither contradicted nor supported”, 5=“very much supported”; answers reverse-coded for analysis) and cognitive emotions (1=“did not feel this at all”, 5=“felt this extremely”). felt during the experience ( $1=$ “did not feel at all”; $5=$ "felt extremely”), embedded within ten emotion items.

Results

Manipulation check

One-way within-subjects ANOVAs with condition as the within-subjects factor confirmed that each emotion was significantly greater within its target condition (all omnibus

24 and pairwise $\left.p s<.001, \eta^{2} s \leq .63\right)$. Ratings for awe and horror were uncorrelated in all conditions, $r s \leq|.10|, p s \geq .23$. 


\section{Writing Prompt Responses}

Awe and Horror differed in all elicitor categories (see Table 2).

[Table 2 near here]

scenarios involved either harm, death or threat without harm. However, among the death/harm/threat situations in Awe, 69\% also contained "silver linings” (e.g., altruistic selfsacrifice, a dying person's positive attitude), indicating that awe elicited by negative experiences often involves positive appraisals. In contrast, $86.9 \%$ of the horror scenarios were elicited by harm/death/threat (e.g., car crash, suicide), $\chi^{2}=149.83, p<.001$, and $0 \%$ contained silver linings, indicating no positive appraisals of horrifying situations. Thus, Hypothesis 3 was supported.

Schema incongruence

Schema incongruence items were reduced using Exploratory Factor Analysis (EFA) with Maximum Likelihood extraction and promax rotation in SPSS. A two-factor structure was observed (see Table 3).[insert footnote 17 here] The first factor was interpreted as Extremity $(\alpha=.84)$ and comprised items describing atypicality and excessive effects. The second factor was interpreted as Spiritual Vastness $(\alpha=.82)$ and comprised items describing expansive perceptions and metaphysical inferences. Standardised regression factor scores were used in subsequent analyses.

[Table 3 near here]

To test Hypothesis 1, one-way within-subjects ANOVAs were conducted on Extremity and Spiritual Vastness with condition (Awe, Horror, Contentment) as the withinsubjects factor. There was a significant main effect for Extremity, $F(2,266)=213.07, p$ $<.001, \eta^{2}=.62$. Post hoc pairwise comparisons with Bonferroni adjustments indicated 
1 Extremity was higher in Horror than in Awe or Contentment, and higher in Awe than

2 Contentment (all ps $<.001$; see Figure 1).

3

4

There was a significant main effect for Spiritual Vastness, $F(1.78,236)=93.08$ with .89 Greenhouse-Geisser correction, $p<.001, \eta^{2}=.41$. Pairwise comparisons indicated Spiritual Vastness was higher in Awe than in Horror or Contentment, and higher in Contentment than Horror (all ps <.001; see Figure 1).

Thus, two types of schema incongruence were observed (Extremity and Spiritual Vastness) and, supporting Hypothesis 1, Extremity was salient in Horror, Spiritual Vastness was salient in Awe and neither were salient in Contentment.

[Figure 1 near here]

NFA

NFA items were reduced using a Maximum Likelihood EFA with promax rotation. Two factors emerged (see Table 4).[insert footnote 18 here] The first factor was interpreted as Shock $(\alpha=.89)$ and comprised items indicating the difficulty in cognitive assimilation. The second factor was interpreted as Chaos $(\alpha=.76)$ and comprised items indicating disorienting mismatches with existing epistemic systems (Neimeyer, Herrero, \& Botella, 2006).

Regression factor scores were used for subsequent analyses.

[Table 4 near here]

To test Hypothesis 2, one-way within-subjects ANOVAs were conducted on Shock and Chaos with condition as the within-subjects factor. There was a main effect for Shock, $F(1.82,242)=154.69$ with .90 Greenhouse-Geisser correction, $p<.001, \eta^{2}=54$. Post hoc Bonferroni pairwise comparisons indicated Shock was higher in Horror than in Awe, $p<.001$ and Contentment, and higher in Awe than Contentment, $p<.008$ (Figure 2).

There was a main effect for Chaos, $F(2,266)=217.43$ with .91 Greenhouse-Geisser correction $, p<.001, \eta^{2}=.65$, and pairwise comparisons indicated Chaos was higher in 
1 Horror than in Awe, $p<.001$, but did not differ between Awe and Contentment, $p=.90$

2 (Figure 2).

3

4 Discussion

5

[Figure 2 near here]

Results from Study 2 indicated that both awe and horror are elicited by schema incongruence and cause greater NFA than contentment. The data also provide further evidence that awe and horror are empirically differentiable based on differences in elicitor and type of schema incongruence.

Qualitative analysis indicated that awe and horror are elicited by different types of experiences. Horror was usually elicited by harmful events, but awe rarely was. Further, awe at harmful events was usually not felt toward the harm itself, but toward something positive occurring despite the harmful situation. Such “silver linings” were completely absent from horror scenarios. Additionally, horror (but not awe) was frequently elicited by information (rather than first-hand witnessing), indicating that "the mere thought of" schema-incongruent harm can elicit horror. In contrast, awe relies more on sensory perception (Shiota et al., 2007). Thus, unlike negative threat-based awe, which has the same elicitors as positive awe (e.g., nature; Gordon et al., 2017) horror had distinctly different elicitors, providing further evidence that horror is not a fearful variant of awe.

Horror and Awe had significantly higher levels of schema incongruence and NFA than Contentment, supporting the hypothesis that awe and horror are schema-incongruent emotional responses. However, awe and horror appear to be differentiable based on what type of schema incongruence is salient: transgressive Extremity in Horror, transcendent Spiritual Vastness in Awe.

The high Chaos in Horror (i.e., confusion due to contradictions of one’s beliefs and values) supports philosophical arguments that horrific things damage systems of meaning. It 
1 also indicates that not all harm elicits horror, only senseless harm that violates our values;

2 morally justifiable harm may fail to horrify us (Rai, Valdesolo, \& Graham, 2017).

3

4

While Horror involved high levels of NFA, Awe did not. Awe showed relatively mild

Shock coupled with and amazement at something that supports schemata (i.e., the inverse of

Chaos). However, because belief-consistent information has greater power to change one's

way of thinking (Fugelsang \& Dunbar, 2005), one possibility is that mildly shocking

experiences that support one's beliefs and values lead to euphoric accommodation (e.g., epiphany, enlightenment).

\section{General discussion}

These studies offer evidence that awe and horror are distinctly different emotional responses to schema incongruence. Awe and horror differed in cognitive appraisals (Study 1), schema-incongruence and elicitors (Study 2). Awe was typically elicited by spiritually vast experiences of nature, people, events and locations, whereas horror was elicited by harmful events and extremity.

Both awe and horror involved NFA, but the NFA in awe was relatively mild, possibly indicating some degree of belief-driven assimilation. Although awe involved NFA (Shock), it also involved moderately high appraisals of certainty (for similar midscale appraisals of certainty, see Gordon et al., 2017; Tong \& Jia, 2017) and amazement at things that support existing values and worldviews (low Chaos), indicating that the experiences were not difficult to assimilate. Previous research similarly found that NFA is either not salient in awe (e.g., not greater in awe relative to a contrast emotion, negatively correlated with awe ratings; Schurtz et al., 2012) or less salient than vastness (e.g., lower ratings, smaller neutral-contrast effect sizes; Chirico et al., 2017; Chirico, Ferrise, Cordella, \& Gaggioli, 2018). The relatively low salience of NFA may be due to the "noetic" sense of revelation and intuitive insight often 
1 reported in awe (James, 1902), which would increase appraisals of certainty (Yaden et al.,

2 2017). Thus, accommodation in awe is not based on schema-conflict resolution, but rather

3 schematic expansion (Shiota et al., 2007), wherein schemata attain a broader scope of

4 meaning and greater explanatory weight (Fugelsang \& Dunbar, 2005).

However, the low NFA in awe raises the question of whether awe involves top-down

6 assimilation. According to Fiedler \& Bless (2000), assimilation involves “appetitive

7 learning” (e.g., exploratory behaviour, self-efficacy, approach motivations) that alters stimuli

8 to match schemata. With this in mind, many of awe's effects indicate schema-driven

9 assimilation rather than stimulus-driven accommodation (Bless \& Fiedler, 2006; Fiedler \&

10 Bless, 2000): awe facilitates inferential abstractions (Valdesolo \& Graham, 2014),

11 strengthens pre-existing beliefs (Valdesolo, Shtulman, \& Baron, 2016), expands "oceanic"

12 categorization (Shiota et al., 2007), increases exploratory behaviour (e.g., using a greater

13 number of unique actions to interact with a novel object; Colantonio \& Bonawitz, 2018) and

14 facilitates creative thinking (Chirico, Glaveanu, Cipresso, Riva, \& Gaggioli, 2018). However,

15 creating unusual or novel connections between semantically distant concepts does not

16 transform or create new schemata. Creativity, imagination and play are assimilative processes

17 that use internal schemata to identify conceptual similarities in external stimuli and shape the

18 stimuli so they more clearly manifest schemata (Piaget, 1962). In contrast, in

accommodation, the external stimulus alters internal cognitive structures so they more accurately reflect stimuli (e.g., imitation, not creativity).

The differences between awe and horror indicate different adaptive functions (Keltner

22 \& Gross, 1999). It has been proposed that awe supports group-level fitness via the downstream social effects of collective bonding, such as broadened social resources (Stellar

24 et al., 2017), prosociality and stabilised social hierarchies (Keltner \& Haidt, 2003). In

25 contrast, horror may facilitate individual survival via its effects on cognition. Horror involves 
1 recognition of painful truths that create a more complex worldview that "inoculates" against

2 psychological malfunction (Janoff-Bulman, 2006) and stress-related impairments of working

3 memory and executive function (Leach, 2012) during subsequent extreme situations. The

4 immediate effects of horror likely include arousal, attentive immobility and intense memory

5 of the elicitor, which enhance learning about dangerous, novel or unpredictable

6 environments, such as those encountered during migration or great ecological or societal

7 change (Diamond \& Zoladz, 2016).

\section{Limitations, contributions and future directions}

Although the studies presented here have shown that the emotional appraisal dimensions and elicitors of horror are different than those in awe, they do not furnish enough evidence to declare that horror qualifies as a distinct emotion. These studies act as first step towards future empirical investigations that can clarify this issue.

One methodological limitation arising from the lack of prior empirical data on horror is that, because its elicitor qualities were unclear, we used retrospective recall to elicit the target emotions, a technique common in emotion research, including research on awe (Shiota et al., 2007; Griskevicius et al. 2010; Gordon et al., 2017), but one that is vulnerable to memory biases (Bradburn et al., 1987). Future studies should use standardized elicitors, such as photos and films, to confirm and expand on the findings presented here. One interesting area of inquiry would be awe and horror differ physiologically. For example, awe involves sympathetic nervous system withdrawal (Shiota et al., 2011), whereas anecdotal reports of horror indicate both sympathetic nervous system activation (e.g., nausea, tachycardia) and autonomic suppression (e.g., tonic immobility; Marx, Forsyth, Gallup, Fusé, \& Lexington, 2008). 
Another limitation of these studies is the use of the emotion labels "awe" and

2 "horror", which may have introduced demand characteristics. In particular, the definition of

3 horror from Study 1 should be tested without emotion labels to ascertain its validity.

4 Although manipulation checks indicated this definition was adequate to differentiate horror

5 from awe, it may require clarification to discriminate horror from other negative emotions,

6 such as defining the elicitor as "shocking” or “against one’s core beliefs or values".

Although Study 2 used a positive emotion contrast condition, there was no negative contrast emotion. Previous studies have investigated how awe differs from multiple positive emotions (Campos et al., 2013; Tong, 2015), but horror requires such clarification, such as how horror differs from fear and disgust.

Finally, the current study only sampled the US population. Although awe has been observed across cultures (Bai et al., 2017), because horror is elicited by irreconcilable contradictions of one's values or beliefs, it may be salient in cultures with analytic ontological beliefs (e.g., the "law" of non-contradiction), such as the English-speaking West, but not in cultures with dialectical ontologies that accept fundamental contradictions (e.g., East Asia; Peng \& Nisbett, 1999).

Despite their limitations, these studies provide valuable data. First, they show that the combination of schema-incongruence and NFA describes not just awe, but also horror, thus introducing the concept of schema-incongruent emotions. Second, they indicate how awe and horror are distinct emotional responses. Third, these studies are (to our knowledge) the first empirical examination of horror as a natural emotion and address a gap in the literature, providing a groundwork for future research. Last, our results problematize the centrality of

23 NFA in awe, which has been theoretically proposed (Keltner \& Haidt, 2003) but not empirically verified. Future research should investigate the nature of accommodation and assimilation in awe. For example, awe may involve a type of NFA that the current measures 
1 could not capture, such as violations of materialist beliefs and naïve physics. Similarly,

2 research should localize which aspects of belief and value systems are violated by horror

3 (e.g., moral imperatives, social trust and conceptualizations of embodiment).

4

5

6 
2 [1]Previous psychological research on horror has been limited to "art horror” (e.g.,

3 psychological responses to cinematic fiction), which is different from "natural horror"

4 towards real-life events (Carroll, 1990).

5 [2]However trauma and horror are not equivalent. Horror is one potential etiological factor in

$6 \quad$ PTSD (Dalgleish \& Power, 2004), but most horrifying events are not traumatic.

7 [3]Because we use the terms "awe" and "horror" to denote three different things (study

8 conditions, Likert emotion ratings, emotions), we use the following conventions to

9 distinguish between them: study conditions are capitalised (Awe, Horror), emotion ratings are

10 italicised and lower-case (awe, horror) and emotions (in discussion, not the data) are

11 unitalicised and lower-case (awe, horror).

12 [4]Data was collected before publication of Gordon et al. (2017), so their findings on

13 “certainty” appraisals did not inform our hypotheses.

14 [5]Because these strict exclusion criteria caused many participants to be excluded, we

15 separately analyzed data from all participants who missed $\leq 2$ attention check questions

$16(\mathrm{~N}=212)$. All results were consistent with those reported here, with no differences in p-

17 values.

18 [6]Prior to data collection, sample sizes were determined using Cohen (1992)'s rule of thumb

19 for medium effect sizes for .05 alpha and doubling it to obviate inattentiveness of online

20 participants.

21 [7]Note that in Study 1, horror was defined as "vast” for participants, which is inconsistent

22 with Study 2's results. When Study 1 was conducted, we assumed schema incongruence in

23 both awe and horror was "vastness". Study 2 tested this assumption, but found no support for

24 it.

25 [8]Study 1 was part of a larger study that included variables not reported here. 
1 [9]Four items were split into two items each: Anticipated Effort-effort ("mental effort",

2 “physical effort”), Situation-Control (“fate/destiny was in control”, “chance/coincidence was

3 in control”), Situation-Responsibility (“fate/destiny was responsible”, “chance/coincidence

4 was responsible”) and Legitimacy-cheated (“you were cheated”, "someone else was

5 cheated”).

6 [10]Similar to Gordon et al. (2017), the reliability for Personal Agency was low, so separate

7 t-tests were conducted on “personal control” and "personal responsibility”. For both

8 individual and composite variables, ratings in Awe were significantly higher than in Horror

$9 \quad(t \mathrm{~s} \leq 3.15, \mathrm{ps}=.002)$.

10 [11]We separately analyzed data from all participants ( $\mathrm{N}=181)$. All results were entirely

11 consistent with those reported, including EFA item loadings and ANOVA results (both

12 omnibus and pairwise).

13 [12]Sample size was based on Everitt (1975), stipulating a N:p ratio of 10.

14 [13]To discriminate elicitation by a person from elicitation by a person's actions, “another

15 person" was defined as elicitation by a person's traits or the fact that such a person exists;

16 elicitation by a person's actions were coded as “events”.

17 [14]“Information” included hearing/reading something second-hand or discovering first-hand 18 evidence (rather than directly witnessing).

19 [15]Due to a database error, twelve horror scenarios were not coded.

20 [16]The two-coder alpha for the "event” category was 0.59 , so a third coder independently

21 coded all scenarios for this theme; the three-coder alpha was .69.

$22[17] \mathrm{KMO}=.835$, Bartlett's $\chi^{2}(91)=2338, p<.001$. Eigenvalues and parallel analysis in SPSS

23 (O’Connor, 2000) indicated two factors. Four items were removed: social significance and 24 personal significance (failed to load), power (double-loaded) and physical size/number 
1 (communality <.20). The two factors showed good discriminant validity, $r=.17$ (i.e., shared $2 \quad 2.9 \%$ of variation).

$3 \quad[18] \mathrm{KMO}=.877$ Bartlett's $\chi^{2}(45)=2235, p<.001$. Eigenvalues and parallel analysis indicated

4 two factors. Three items were removed: absorbed (communality <.20), shocked (cross-

5 loaded), easy to understand (cross-loaded). Amazement cross-loaded, but was retained

6 because the loading difference was almost .40. The two factors were negatively correlated (.7 49), sharing $24 \%$ of variance. 


\section{References}

2 Armstrong, T., \& Detweiler-Bedell, B. (2008). Beauty as an emotion: The exhilarating prospect of mastering a challenging world. Review of General Psychology, 12(4), 305329.

Bai, Y., Maruskin, L., Chen, S., Gordon, A., Stellar, J., McNeil, G., ... Keltner, D. (2017). Awe, the diminished self, and collective engagement: Universals and cultural variations in the small self. Journal of Personality and Social Psychology, 113(2), 185-209.

Berenbaum, H. (2002). Varieties of joy-related pleasurable activities and feelings. Cognition \& Emotion, 16(4), 473-494.

Bless, H., \& Fiedler, H. (2006). Mood and the regulation of information processing and behavior. In J. P. Forgas (Ed.), Affect in social thinking and behavior (pp. 65-84). New

Bonner, E., \& Friedman, H. (2011). A conceptual clarification of the experience of awe: An interpretative phenomenological analysis. The Humanistic Psychologist, 39, 222-235.

Brewin, C., \& Holmes, E. (2003). Psychological theories of posttraumatic stress disorder. Clinical Psychology Review, 23(3), 339-376.

Brewin, C., \& Shapiro, D. (1984). Beyond locus of control: Attribution of responsibility for positive and negative outcomes. British Journal of Psychology, 75(1), 43-49.

Campos, B., Shiota, M., Keltner, D., Gonzaga, G., \& Goetz, J. (2013). What is shared, what is different? Core relational themes and expressive displays of eight positive emotions. Cognition \& Emotion, 27(1), 37-52.

22 Carroll, N. (1990). The philosophy of horror: Or, paradoxes of the heart. Cambridge: Routledge.

Cavell, S. (1979). The claim of reason: Wittgenstein, skepticism, morality, and tragedy. New York: Oxford University Press. 
1 Chirico, A., Cipresso, P., Yaden, D., Biassoni, F., Riva, G., \& Gaggioli, A. (2017).

2 Effectiveness of immersive videos in inducing awe: An experimental study. Scientific

3 Reports, 7(1).

4 Chirico, A., Ferrise, F., Cordella, L., \& Gaggioli, A. (2018). Designing awe in virtual reality: An experimental study. Frontiers in Psychology, 8, 2351.

6 Chirico, A., Glaveanu, V., Cipresso, P., Riva, G., \& Gaggioli, A. (2018). Awe enhances creative thinking: An experimental study. Creativity Research Journal, 30(2), 123-131.

8 Clore, G., \& Gasper, K. (2000). Feeling is believing: Some affective influences on belief. In N. Frijda, A. Manstead, \& S. Bem (Eds.), Emotions and beliefs: How feelings influence thoughts (pp. 10-44). Cambridge University Press.

11 Cohen, J. (1992). A power primer. Psychological Bulletin, 112(1), 155-159.

12 Colantonio, J., \& Bonawitz, E. (2018). Awesome play: Awe increases preschooler's exploration and discovery. Open Science Framework.

Dalgleish, T., \& Power, M. (2004). Emotion-specific and emotion-non-specific components of posttraumatic stress disorder (PTSD): Implications for a taxonomy of related psychopathology. Behaviour Research and Therapy, 42(9), 1069-1088.

Darwin, C. (1872). The expression of the emotions in man and animals. London: HarperCollins.

Diamond, D., Campbell, A., Park, C., Halonen, J., \& Zoladz, P. (2007). The temporal basis of stress-induced amnesia, flashbulb and traumatic memories, and the YerkesDodson Law. Neural Plasticity, 60803, 1-33.

Diamond, D., \& Zoladz, P. (2016). Dysfunctional or hyperfunctional? The amygdala in posttraumatic stress disorder is the bull in the evolutionary china shop. Journal of Neuroscience Research, 94(6), 437-444. 
1 Everitt, B. (1975). Multivariate analysis: The need for data, and other problems. The British 2 Journal of Psychiatry, 126(3), 237-240.

3 Faul, F., Erdfelder, E., Lang, A., \& Buchner, A. (2007). G*Power 3: A flexible statistical

4 power analysis program for the social, behavioral, and biomedical sciences. Behavior Research Methods, 39(2), 175-191.

Feigenson, N. (2002). Emotions, risk perceptions and blaming in 9/11 cases. Brooklyn Law Review, 68(4), 959-1001.

Fiedler, K., \& Bless, H. (2000). The formation of beliefs at the interface of affective and cognitive processes. In N. Frijda, A. Manstead, \& S. Bem (Eds.), Emotions and beliefs: How feelings influence thoughts (pp. 144-170). Paris: Cambridge University Press.

Fiske, S., \& Linville, P. (1980). What does the schema concept buy us? Personality and Social Psychology Bulletin, 6(4), 543-557.

Fugelsang, J., \& Dunbar, K. (2005). Brain-based mechanisms underlying complex causal thinking. Neuropsychologia, 43, 1204-1213.

Gordon, A., Stellar, J., Anderson, C., Mcneil, G., Loew, D., \& Keltner, D. (2017). The dark side of the sublime: Distinguishing a threat-based variant of awe. Journal of Personality and Social Psychology, 113(2), 310-328.

Gutschick, V., \& BassiriRad, H. (2003). Extreme events as shaping physiology, ecology, and evolution of plants: Toward a unified definition and evaluation of their consequences. New Phytologist, 160, 21-42.

James, W. (1902). The varieties of religious experience. London: Longmans, Green.

Janoff-Bulman, R. (1992). Shattered assumptions: Toward a new psychology of trauma. New York: Free Press.

Janoff-Bulman, R. (2006). Schema-change perspectives on posttraumatic growth. In L. 
practice (pp. 81-99). Mahwah, NJ: Lawrence Erlbaum Associates.

2 Kant, I. (1951). Critique of Judgment. (J.H. Bernard, Trans.). New York: Hafner Publishing Company. (Original work published 1790).

Keltner, D., \& Gross, J. (1999). Functional accounts of emotions. Cognition and Emotion, 13(5), 467-480.

6 Keltner, D., \& Haidt, J. (2003). Approaching awe, a moral, spiritual, and aesthetic emotion. Cognition and Emotion, 17(3), 297-314.

Krause, N., \& Hayward, R. (2015). Assessing whether practical wisdom and awe of God are associated with life satisfaction. Psychology of Religion and Spirituality, 7(1), 51-59.

Kristeva, J. (1982). Powers of Horror: An Essay on Abjection. (Trans. L.S. Roudiez.). New York: Columbia University Press.

Leach, J. (1994). Survival psychology. Basingstoke: MacMillan.

Leach, J. (2012). Maladaptive behavior in survivors: Dysexecutive survivor syndrome. Aviation Space and Environmental Medicine, 83(12), 1152-1161.

Lodge, M., \& Taber, C. (2000). Three steps toward a theory of motivated political reasoning. In A. Lupia, M. D. (Mathew D. McCubbins, \& S. L. Popkin (Eds.), Elements of reason: Cognition, choice, and the bounds of rationality (pp. 183-213). London: Cambridge University Press.

Marx, B., Forsyth, J., Gallup, G., Fusé, T., \& Lexington, J. (2008). Tonic immobility as an evolved predator defense: Implications for sexual assault survivors. Clinical Psychology: Science and Practice, 15(1), 74-90.

McCann, L., \& Pearlman, L. (1990). Vicarious traumatization: A framework for understanding the psychological effects of working with victims. Journal of Traumatic Stress, 3(1), 131-149. 
psychological adaptation. The Counseling Psychologist, 16(4), 531-594.

Miller, A. (2004). Introduction and Overview. In A. Miller (Ed.), The Social Psychology of Good and Evil (pp. 1-17). New York: Guilford.

Neimeyer, R., Herrero, O., \& Botella, L. (2006). Chaos to coherence: Psychotherapeutic integration of traumatic loss. Journal of Constructivist Psychology, 19(2), 127-145.

Nisbett, R., \& Wilson, T. (1977). Telling more than we can know: Verbal reports on mental processes. Psychological Review, 84(3), 231-259.

O’Connor, B. (2000). SPSS and SAS programs for determining the number of components using parallel analysis and Velicer’s MAP test. Behavior Research Methods, Instruments, \& Computers, 32(3), 396-402.

Peng, K., \& Nisbett, R. (1999). Culture, dialectics, and reasoning about contradiction. American Psychologist, 54(9), 741-754.

Piaget, J. (1950). The psychology of intelligence. London: Routledge \& Paul.

Piaget, J. (1962). Play, dreams and imitation. New York: Norton.

Piff, P., Dietze, P., Feinberg, M., Stancato, D., \& Keltner, D. (2015). Awe, the small self, and prosocial behavior. Journal of Personality and Social Psychology, 108(6), 883-899.

Rai, T., Valdesolo, P., \& Graham, J. (2017). Dehumanization increases instrumental violence, but not moral violence. PNAS, 114(32), 8511-8516.

Scherer, K. (2005). What are emotions? And how can they be measured? Social Science Information, 44(4), 695-729.

Schurtz, D., Blincoe, S., Smith, R., Powell, C., Combs, D., \& Kim, S. (2012). Exploring the social aspects of goose bumps and their role in awe and envy. Motivation and Emotion, 36(2), 205-217.

Shiota, M., Keltner, D., \& Mossman, A. (2007). The nature of awe: Elicitors, appraisals, and effects on self-concept. Cognition \& Emotion, 21(5), 944-963. 
1 Silvia, P. (2010). Confusion and interest: The role of knowledge emotions in aesthetic

2

3

4 experience. Psychology of Aesthetics, Creativity, and the Arts, 4(2), 75-80.

Singer, M. (2004). The Concept of Evil. Philosophy, 78(308), 185-214.

Smith, C., \& Ellsworth, P. (1985). Patterns of cognitive appraisal in emotion. Journal of Personality \& Social Psychology, 48(4), 813-838.

Stellar, J., Gordon, A., Piff, P., Cordaro, D., Anderson, C., Bai, Y., ... Keltner, D. (2017). Self-transcendent emotions and their social functions: Compassion, gratitude, and awe bind us to others through prosociality. Emotion Review, 9(3), 200-207.

Taylor, S., \& Fiske, S. (1978). Salience, attention, and attribution: Top of the head phenomena. Advances in Experimental Social Psychology, 11, 249-288.

Tong, E., \& Jia, L. (2017). Positive emotion, appraisal, and the role of appraisal overlap in positive emotion co-occurrence. Emotion, 17(1), 40-54.

Valdesolo, P., \& Graham, J. (2014). Awe, uncertainty, and agency detection. Psychological Science, 25(1), 170-178.

Valdesolo, P., Shtulman, A., \& Baron, A. (2016). Science is awe-some: The emotional antecedents of science learning. Emotion Review, 1-23.

Van Cappellen, P., \& Saroglou, V. (2012). Awe activates religious and spiritual feelings and behavioral intentions. Psychology of Religion and Spirituality, 4(3), 223-236.

Van Cappellen, P., Saroglou, V., Iweins, C., Piovesana, M., \& Fredrickson, B. (2013). Selftranscendent positive emotions increase spirituality through basic world assumptions. Cognition and Emotion, 27(8), 1378-1394.

Wierzbicka, A. (1988). The semantics of emotions: Fear and its relatives in English. Australian Journal of Linguistics, 10, 359-375.

Yaden, D., Haidt, J., Hood, R., Vago, D., \& Newberg, A. (2017). The varieties of selftranscendent experience. Review of General Psychology, 21(2), 143-160. 
1 Yaden, D., Le Nguyen, K., Kern, M., Wintering, N., Eichstaedt, J., Schwartz, H., ...

2 Newberg, A. (2017). The noetic quality: A multimethod exploratory study. Psychology

3 of Consciousness: Theory, Research and Practice, 4(1), 54-62.

4 Yum, Y.-O., \& Schenck-Hamlin, W. (2005). Reactions to 9/11 as a function of terror 286.

7

8 